\title{
Feasibility and Acceptability of a Web-Based, Gender-Specific Intervention for Women with Substance Use Disorders
}

\author{
Dawn E. Sugarman, PhD, ${ }^{1-3}$ Laurel E. Meyer, BA, ${ }^{1,2}$ Meghan E. Reilly, BA, ${ }^{1,2}$ \\ and Shelly F. Greenfield, MD, MPH ${ }^{1-3}$
}

\begin{abstract}
Background: Women face unique issues related to their substance use. Treatment programs that incorporate gender-specific components can to lead to enhanced outcomes for women with substance use disorders (SUDs). Nevertheless, most women receive treatment in mixed-gender SUD treatment programs that lack womenspecific components. Programs cite lack of expertise and staff time as barriers to providing gender-specific care. Leveraging technology can address some of the challenges of providing gender-specific care in a mixed-gender setting by offering an accessible, cost-effective alternative to in-person services.

Materials and Methods: We developed a gender-specific, web-based, psychoeducational intervention for women with SUDs as an addition to treatment as usual in a mixed-gender SUD treatment program. Next, we examined the feasibility and acceptability of this single-session intervention in a prepilot study with 30 women with SUDs. Based on these data, we refined the intervention and tested feasibility and acceptability with 60 women with SUDs.

Results: Participants indicated a high level of satisfaction with the intervention. Satisfaction did not significantly differ by level of care (inpatient, partial hospitalization, or outpatient) or age, and was not associated with the number of previous treatment episodes. Participants highlighted the importance of addressing co-occurring disorders, trauma, and sexual abuse in SUD treatment for women.

Conclusions: Women in this study found this web-based gender-specific intervention relevant regardless of their previous experience in treatment. Delivery of this intervention is feasible and has the potential to be a highly sustainable strategy for increasing exposure to gender-specific SUD components of care.
\end{abstract}

Keywords: women, substance use disorders, technology, gender-specific

\section{Introduction}

$\mathbf{T}$ HERE ARE SIGNIFICANT sex differences in the epidemiology, physiology, and treatment course of substance use disorders (SUDs). ${ }^{1-4}$ Given these differences, there has been a growing interest in gender-specific treatment for women with SUDs. 5 The term "gender-specific" refers to treatment that incorporates factors that are more prevalent in women that may affect their treatment outcomes, including trauma, co-occurring psychiatric disorders, relationships with children and intimate partners, and physical health issues. ${ }^{6,7}$
The literature on gender-specific treatment is mixed. Quasiexperimental research has found that compared with mixed-gender treatment, single-gender treatment for women with SUDs is associated with lower criminal activity and incarceration, ${ }^{8-10}$ lower rates of substance use,, 11 and increased continuity of care following discharge. ${ }^{12}$ Of note, single-gender treatment may not necessarily include genderspecific program elements beyond the women-only environment, and some evidence suggests that a single-gender environment in the absence of gender-specific content is not sufficient to affect treatment outcomes for women. ${ }^{13}$

\footnotetext{
${ }^{1}$ Division of Alcohol and Drug Abuse, McLean Hospital, Belmont, Massachusetts.

${ }^{2}$ Division of Women's Mental Health, McLean Hospital, Belmont, Massachusetts.

${ }^{3}$ Department of Psychiatry, Harvard Medical School, Boston, Massachusetts.

Portions of this article were presented in poster format at the 50th annual convention of the Association for Behavioral and Cognitive Therapies, October 2016, New York, NY and the 51st annual convention of the Association for Behavioral and Cognitive Therapies, November 2017, San Diego, CA.
} 
Randomized controlled trials (RCTs) have examined genderspecific treatments for subpopulations of women with SUDs, including, women with co-occurring disorders, ${ }^{14,15}$ women in the criminal justice system, ${ }^{16}$ and women with children. ${ }^{17}$ The majority of these studies have been small pilot studies with a standard treatment comparison group,${ }^{15-17}$ with only one study finding an effect of the treatment on substance use. ${ }^{17}$ Larger RCTs have compared gender-specific treatment with gender-focused psychoeducation ${ }^{14}$ and gender-neutral treatment, ${ }^{18}$ with both studies finding equivalent substance use outcomes for treatment and control groups. ${ }^{18}$

The only study that has not focused on a subpopulation of women is the Women's Recovery Group (WRG) study. The WRG is a gender-specific group treatment for women with SUDs designed for women heterogeneous with respect to their substance of use, and demographic and clinical characteristics, to reflect the diverse characteristics of treatmentseeking women with SUDs. ${ }^{19}$ The WRG was examined in two RCTs (a stage I and a multisite stage II trial) ${ }^{20,21}$ in comparison with mixed-gender group drug counseling (GDC). In both trials, women in the WRG and GDC demonstrated clinically relevant reductions in substance use after 12 weeks of group treatment. ${ }^{20,21}$ In the stage I trial, only the women in the WRG continued to show these reductions in substance use 6 months posttreatment. ${ }^{20}$

A review of SUD treatment programs in the United States found that only $31 \%$ have gender-specific components. ${ }^{22}$ Women-only treatment programs are more likely to include gender-specific components ${ }^{6,23}$; however, these programs are limited in number, and the majority of women receive treatment in mixed-gender settings. ${ }^{5,24}$ To address this important issue, the Substance Abuse and Mental Health Services Administration (SAMHSA) created recommendations for including gender-specific components of care in mixedgender settings. ${ }^{25}$ However, the impact of existing genderspecific treatments is restricted by in-person delivery requiring intensive clinician training thereby limiting dissemination and implementation. Fewer women relative to men in mixedgender settings can be an additional barrier. There is a paucity of research demonstrating effective strategies to implement this care for women in mixed-gender settings.

Digital interventions have the potential to address some of the treatment challenges of providing gender-specific care in a mixed-gender setting by offering an accessible, costeffective alternative to in-person services. ${ }^{26}$ Web-based interventions can be widely disseminated and used in a number of treatment settings, as well as home or public settings with an internet connection. ${ }^{27,28}$ Digital interventions are also a helpful solution for women who lack childcare or transportation, have scheduling conflicts with outpatient services, or are not ready to seek traditional services due to stigma or social anxiety. ${ }^{28}$ Digital interventions can be customized to meet the needs of users and offer an individualized experience that could offset the heavy reliance on group treatments that often dominate SUD treatment programs. ${ }^{29}$

Several studies support the effectiveness of digital interventions for SUDs. ${ }^{30-34}$ There is also evidence that women seek out health-related information on the internet more than men $^{35,36}$; thus, a digital intervention may be particularly appealing for women. In support of this, when gender differences were examined in a study of a computer-assisted intervention for SUDs, ${ }^{37}$ results showed that although gender did not moderate treatment outcome, women had higher acceptability ratings of the intervention than men; in turn, acceptability was positively associated with abstinence in women, but not men. Research in digital interventions with women-only samples is limited to a series of studies of a computerized screening and brief intervention (e-SBI) targeting postpartum women who self-reported illicit drug use. ${ }^{38-40}$ Although results supported short-term efficacy on postpartum drug use, this e-SBI was not designed to address a treatment population of women with SUDs.

One promising enhancement to treatment is the use of psychoeducational material as a low-cost, nonburdensome, effective add-on component. There is some evidence to support the use of women-specific psychoeducational material. Women in the WRG rated the gender-specific information that they received in group as "very helpful". ${ }^{21}$ In a large multisite trial, women's health psychoeducation delivered in group format performed as well as trauma-focused behavioral therapy in reducing posttraumatic stress disorder (PTSD) symptoms for women with co-occurring SUDs and PTSD. ${ }^{14}$ However, these results need to be interpreted with caution because of the lack of a no-treatment or minimaltreatment control group. In addition to therapist-delivered models, studies have demonstrated the feasibility of delivering psychoeducation for a variety of mental health problems in a digital format. ${ }^{41-43}$ For example, digital platforms of psychoeducation have been effective in reducing symptom severity in individuals with borderline personality disorder ${ }^{41}$ and mental health symptoms in women veterans, ${ }^{42}$ and in symptom and knowledge improvement for individuals with schizophrenia. ${ }^{43}$

The goal of this study was to develop a web-based, genderspecific intervention (GSI) as an addition to treatment as usual for women with SUDs. In a prepilot study, we examined feasibility and acceptability of the GSI for women enrolled in mixed-gender inpatient treatment for SUDs. These data were used to refine the GSI, which was then tested in a pilot study for women enrolled in various levels of mixedgender treatment for SUDs (inpatient, partial hospitalization, and outpatient). In both studies, we assessed attitudes regarding the importance of gender-specific topics, satisfaction, and the relevance of content areas included in the GSI.

\section{Materials and Methods}

\section{Overview}

The prepilot and pilot studies used the same measures and general procedures. Modifications were made to the GSI from the prepilot to pilot study based on participant feedback.

\section{Procedure}

The protocols for both studies received approval from the Partners HealthCare Institutional Review Board, and written informed consent was obtained from participants. All participants were recruited from McLean Hospital's Alcohol and Drug Abuse Treatment Program (Belmont, MA). When introducing the study to eligible participants, the research assistant (RA) explained that the study was assessing the feasibility and satisfaction of a new digital program for women with SUDs. Women who enrolled in the study met with the RA to complete study procedures, including, baseline 
assessments, the GSI, and postintervention questionnaires on an iPad.

The GSI was designed as a single-session intervention; however, we allowed participants to complete it in two visits if needed. Two participants (one prepilot, one pilot) needed to complete the GSI in two sessions due to competing treatment demands (e.g., meetings with treatment providers or groups). Participants recruited from inpatient completed the GSI while in treatment on the inpatient unit, which has an average stay of 4 days. All inpatient participants were required to have been on the inpatient unit for $>24$ hours before the RA approached them about the study. Participants recruited from partial hospitalization or outpatient treatment could complete the study at any point during their treatment. The average length of stay in partial hospitalization is 2 weeks. We did not collect information on how long participants had been in outpatient treatment when they completed the study.

Participants were compensated with a $\$ 25$ gift card upon completion of the study. Participants who completed the study across two visits were compensated a $\$ 10$ gift card at their first visit and a $\$ 15$ gift card upon completion of their second visit. Participants were informed during the consent process that they could discontinue the study at any time and they would be compensated for the portion of the study they completed.

\section{Materials}

GSI development. In the initial development, psychoeducational material from three modules of the $\mathrm{WRG}^{19-21}$ was modified from a group therapy format to a patient-centered, web-based format. Material from the WRG is based on cognitive behavioral principles and relapse prevention. Three topics from the WRG that are of key relevance to women with
SUDs were chosen for adaptation to a digital platform: (1) The Effect of Drugs and Alcohol on Women's Health, (2) Managing Mood, Anxiety, and Eating Problems without Using Substances, and (3) Women and their Partners. Each topic was converted into one module of the GSI. In the WRG, these topics are presented in group format by the therapist in an interactive style with open discussion among women participants. Modifications for the web-based format focused on presenting materials in an engaging style that could be understood by women of varying literacy levels. Each module begins with an overview of the content, provides visual and text information on the topic, and includes "Knowledge Check" questions to keep participants engaged with the material (Fig. 1). Each module ends with "Take-Home Messages" that summarize key concepts (Fig. 2).

\section{Measures}

Basic demographic information was collected Pre-intervention in the prepilot and pilot studies. Additional questions regarding primary substance of use, treatment history, and psychiatric problems were collected in the pilot study only. Both the prepilot and pilot studies otherwise used the same measures.

The gender-specific program assessment, adapted for this study based on a measure that has been previously used to determine the level of gender-responsivity in mixed-gender programs, ${ }^{5,10}$ was completed preintervention to measure participants' ratings of the importance of the inclusion of 10 gender-specific topics as part of SUD treatment. Ratings were assessed with a Likert scale of $0-4(0=$ not at all important; $4=$ extremely important). Examples of items include: "sexual abuse," "the role of co-occurring psychiatric disorders in recovery," and "community support for women."
FIG. 1. Screenshot of knowledge check question in the genderspecific intervention (GSI).

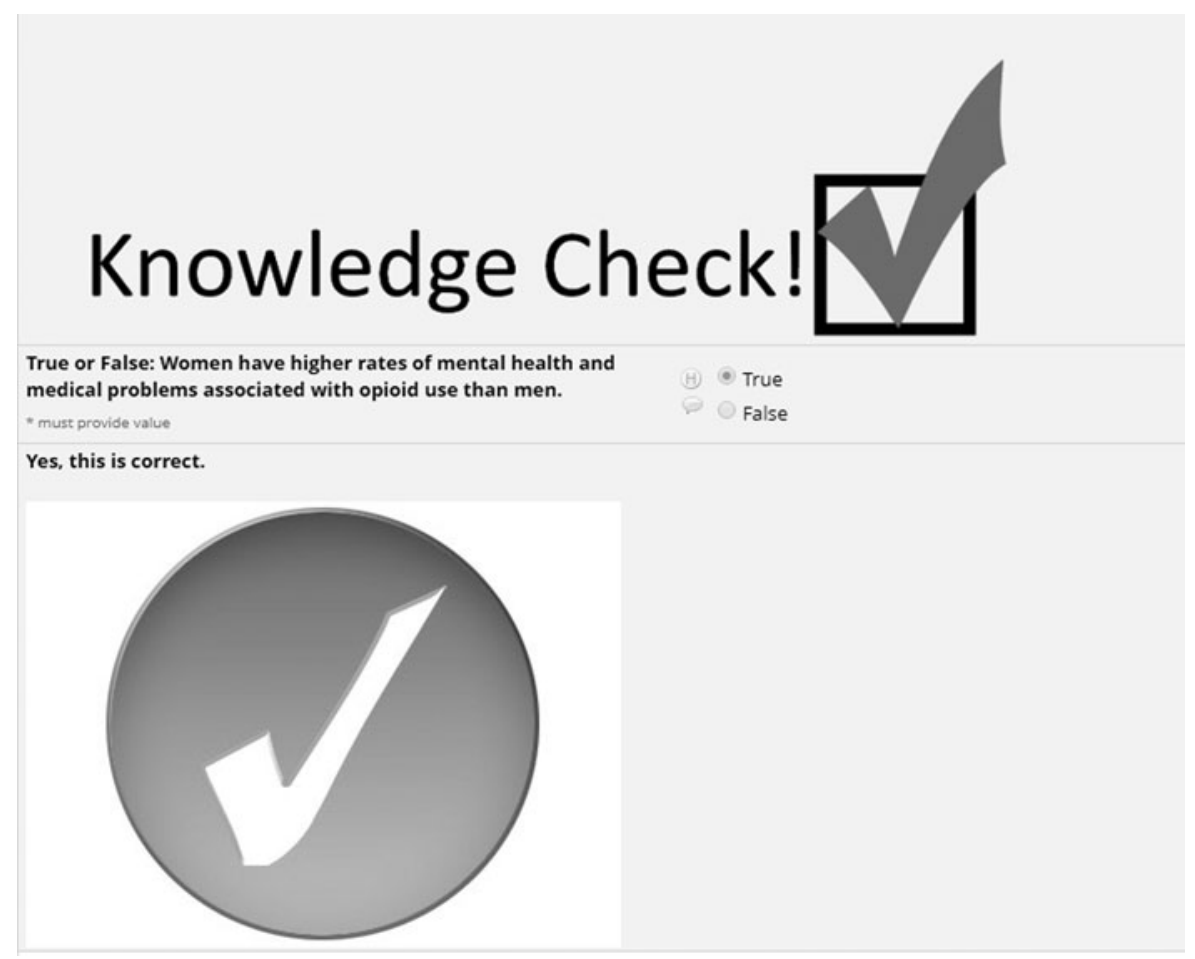




\section{Take Home Message}

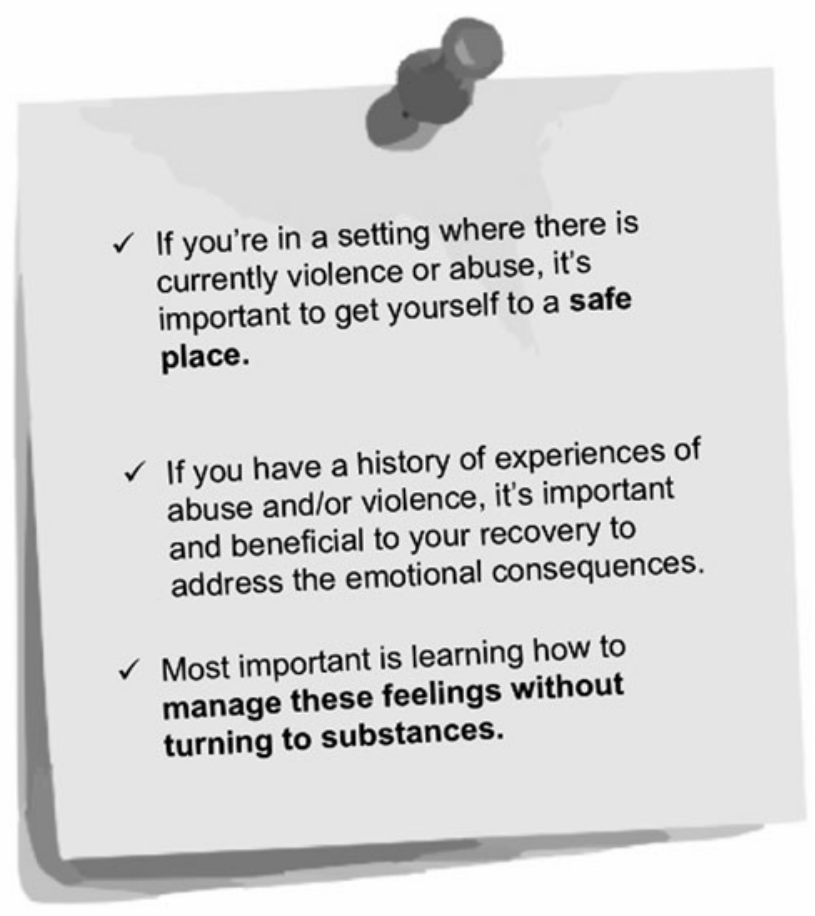

FIG. 2. Screenshot of take-home message in the GSI.

Participants were given the option to list additional topics of importance for treatment for SUDs.

The Relevance Questionnaire was developed to assess participants' perceptions of how relevant 15 content areas of the GSI were to their recovery. Post-intervention, participants rated each item using a Likert scale of $0-4(0=$ not at all relevant; $4=$ extremely relevant). Sample items include: "the effects of substance use on self-care" and "the influence of a partner on recovery." Participants were also asked to list additional topics of relevance.

A modified version of the Client Satisfaction Questionnaire $(\mathrm{CSQ})^{20,44}$ was administered postintervention to measure participants' satisfaction with features of the GSI. The CSQ contains 10 items, which are summed with scores ranging from 0 to 40 , with 40 being the most satisfied. A sample item includes: "Were you satisfied with the type of information you received regarding women and substance use problems," which was answered on a four-point Likert scale of "very satisfied" to "quite dissatisfied." Five additional items were added to this questionnaire: four to assess satisfaction with the technological components of the intervention, and one to ask participants if they would make any changes to the GSI. For example, participants answered the question "How visually appealing was the computerized program" on a four-point Likert scale from "extremely visually appealing" to "not at all visually appealing." Frequencies were used to examine the results of these additional five questions.

\section{Qualitative feedback}

Qualitative feedback on the GSI was collected from responses to open-ended items on the questionnaires, as well as verbal comments made during the intervention. On the Relevance Questionnaire, participants were asked to "list any additional topics that you think would be helpful to include in a computerized program for women with substance use disorders." On the CSQ, participants were instructed to "provide additional comments or suggestions on the topics you reviewed or your experience with the computerized program." In addition, participants who responded "yes" to an item on the CSQ asking if they would make any changes to the GSI, were asked to explain what changes they would make. Lastly, the RA wrote down any verbal comments that the participants made as they were completing the GSI.

\section{Prepilot Study}

\section{Methods}

Participants. Participants $(n=30)$ for the prepilot study were recruited from an inpatient detoxification unit that offers mixed-gender treatment for individuals with SUDs, including, group therapy, individual counseling, and medical detoxification. The inpatient treatment program does not currently have a gender-specific component of care. Women were eligible to participate if they were 18 years of age or older. Women were excluded from the study if they (1) had 
an acute psychiatric or medical condition that would impair them from giving informed consent and completing study procedures, and (2) were admitted to the unit involuntarily. Fifty women were identified by clinicians as eligible for the study during the recruitment period. Of those 50, $30(60 \%)$ were enrolled, $14(28 \%)$ were discharged before the RA could approach them, $5(10 \%)$ said they were too busy to participate in the study, and $1(2 \%)$ was not interested in participating.

\section{Results}

Demographics and background characteristics. Table 1 provides a summary of the demographic characteristics for the sample, which was predominantly white and well educated. Half of the sample had children, with an average of 2.1 children (standard deviation $[\mathrm{SD}]=1.3$; range $1-4$ ). Thirteen women $(43 \%)$ reported that they had a current intimate partner that used alcohol or drugs. Few participants had ever participated in a women-only treatment program for SUDs (23\%); however, $50 \%$ of participants had attended womenonly self-help meetings.

Gender-specific program assessment. Before engaging with the GSI, the three components that were rated highest in importance for an SUD program to address were: the role of co-occurring psychiatric disorders in recovery, trauma and PTSD and sexual abuse (Table 2). With the exception of one component (spiritual needs, mean $=2.9, \mathrm{SD}=1.0$ ), average ratings for all topics were above 3 (i.e., "very important").

Completion and satisfaction. All the women who participated in the prepilot study completed all three modules of the GSI and accompanying assessments. Time spent on each module ranged from 6.2 minutes $(\mathrm{SD}=3.9$; Managing Mood, Anxiety and Eating Disorders) to 7.7 minutes $(\mathrm{SD}=3.8$; The Effects of Drugs and Alcohol on Women's Health); the average total completion time for the GSI was 26.6 minutes $(\mathrm{SD}=14.2)$. Each of the content areas were rated as above average in relevance with the mean ranging from 2.5 to 3.7 (Table 3). The top three rated content areas were: the effects of substance use on self-care, the link between substance use and other mental health problems, and the symptoms of depression. The lowest rated content area was the effects of marijuana on women's health, with $40 \%$ of participants rating it as "neutral," "a little," or "not at all" relevant.

The mean satisfaction score on the CSQ was 34.8 ( $\mathrm{SD}=4.4$; range $22-40$ ) out of 40 , indicating that participants were highly satisfied with the intervention. Of note, $97 \%$ of participants indicated that the gender-specific information was "extremely" (60\%), "very" (30\%), or "a little" (6.7\%) helpful. With regard to technological aspects, $93 \%$ of participants rated the GSI as "extremely easy to use," and all participants rated the GSI as "somewhat" to "extremely" visually appealing. Moreover, 97\% of participants reported

Table 1. Prepilot and Pilot Sample Demographic Characteristics

\begin{tabular}{|c|c|c|}
\hline & Prepilot $(\mathrm{n}=30)$ & Pilot $(\mathrm{n}=60)$ \\
\hline Age, years, mean (SD) & 39.2 (13.9) & $39.7(15.0)$ \\
\hline Range & $20-68$ & $18-74$ \\
\hline \multicolumn{3}{|l|}{ Race, $n(\%)$} \\
\hline White & $29(96.7)$ & $55(91.7)$ \\
\hline Black or African American & $1(3.3)$ & 0 \\
\hline Asian & $1(3.3)$ & $2(3.3)$ \\
\hline American Indian/Alaskan Native & 0 & $1(1.7)$ \\
\hline \multicolumn{3}{|l|}{ Ethnicity, $n(\%)$} \\
\hline Not Hispanic or Latino & $27(90.0)$ & $56(93.3)$ \\
\hline \multicolumn{3}{|l|}{ Marital status, $n(\%)$} \\
\hline Married/living with partner & $5(16.6)$ & $19(31.6)$ \\
\hline Widowed & $1(3.3)$ & $2(3.3)$ \\
\hline Divorced/separated & $9(30.0)$ & $13(21.7)$ \\
\hline Never married & $15(50.0)$ & $26(43.3)$ \\
\hline \multicolumn{3}{|l|}{ Educational attainment, $n(\%)$} \\
\hline High school or less & $5(16.6)$ & $7(11.7)$ \\
\hline Some college or more & $25(83.3)$ & $53(88.3)$ \\
\hline \multicolumn{3}{|l|}{ Occupational status, $n(\%)$} \\
\hline Employed full time & $6(20.0)$ & $15(25.4)$ \\
\hline Employed part time & $3(10.0)$ & $13(22.1)$ \\
\hline Unemployed & $12(40.0)$ & $20(33.9)$ \\
\hline Retired & 0 & $6(10.0)$ \\
\hline Disability & $8(26.7)$ & $4(6.7)$ \\
\hline Student & $1(3.3)$ & $1(1.7)$ \\
\hline \multicolumn{3}{|l|}{ Sexual orientation, $n(\%)$} \\
\hline Heterosexual/straight & $25(83.3)$ & $54(90.0)$ \\
\hline Bisexual & $4(13.3)$ & $4(6.7)$ \\
\hline Questioning/unsure & 0 & $1(1.7)$ \\
\hline
\end{tabular}

$\mathrm{SD}$, standard deviation. 
Table 2. Importance of Addressing Gender-Specific Topics in Treatment For Substance Use Disorders, Mean (SD)

\begin{tabular}{lcc}
\hline & Prepilot $(\mathrm{n}=30)$ & Pilot $(\mathrm{n}=60)$ \\
\hline The role of co-occurring psychiatric disorders in recovery & $3.7(0.5)$ & $3.6(0.6)$ \\
Trauma and posttraumatic stress disorder & $3.6(0.7)$ & $3.2(1.0)$ \\
Sexual abuse & $3.6(0.7)$ & $3.1(1.2)$ \\
Counseling about healthy relationships & $3.5(0.5)$ & $3.4(0.7)$ \\
Family violence & $3.5(0.8)$ & $3.0(1.2)$ \\
Community support for women & $3.4(0.7)$ & $3.2(0.9)$ \\
The role of parenting/caretaking in recovery & $3.3(0.7)$ & $3.2(1.0)$ \\
Housing needs & $3.1(1.1)$ & $3.0(1.2)$ \\
Women's sexuality and substance use & $3.1(1.0)$ & $2.8(1.3)$ \\
Spiritual needs & $2.9(1.0)$ & $2.6(1.1)$ \\
\hline
\end{tabular}

that the knowledge check questions were helpful, with $40 \%$ indicating they helped "somewhat" and 57\% endorsing that they helped "a great deal." Forty-three percent of participants reported that they would have liked more interactive questions throughout the GSI.

Qualitative feedback from participants. Seventeen participants $(57 \%)$ provided qualitative feedback during the prepilot study, with some providing feedback at multiple points: 7 participants $(23 \%)$ provided topic suggestions on the Relevance Questionnaire, 6 participants (20\%) included additional comments/suggestions on the CSQ, 7 participants $(23 \%)$ explained changes they would make to the program on the CSQ, and 11 participants (37\%) made verbal comments during completion of the GSI. Responses were reviewed independently by the principal investigator (PI) and RA and grouped by "comments" or "suggestions," and each group was reviewed for themes. The PI and RA reviewed the themes together and either differentiated them further or grouped them according to commonalities. Quotes were se- lected that best highlight the given theme. Three themes emerged from the comments, including:

(1) The importance of learning the gender-specific information:

"...just learning all of this information is crucial for all women. I was unaware of a lot of it."

(2) Relating to the information in the GSI:

"I feel like [the] survey was right on and explained my problem as a woman with addiction to the T."

(3) Delivery of the information:

"It is really helpful to see the information in this way. In groups, it is great to be able to discuss these things with other people but it is easy to miss something, so having it all laid out on an iPad like this is really helpful to remember everything and also see it visually."

Suggestions for changes to the program were categorized into four themes: (1) adding information on trauma, (2) providing more in-depth information on depression, (3) expanding on the role of relationships in recovery, and (4) increasing the visual/aesthetic elements of the GSI.

Table 3. Relevance of Content Areas of the Gender-Specific Intervention, Mean (SD)

\begin{tabular}{|c|c|c|}
\hline & Prepilot $(\mathrm{n}=30)$ & Pilot $(\mathrm{n}=60)$ \\
\hline The effects of substance use on self-care & $3.7(0.5)$ & $3.4(0.8)$ \\
\hline The link between substance use and other mental health problems & $3.6(0.6)$ & $3.6(0.7)$ \\
\hline The symptoms of depression & $3.6(0.6)$ & $3.4(0.9)$ \\
\hline The symptoms of anxiety & $3.5(0.9)$ & $3.6(0.7)$ \\
\hline The influence of a partner on recovery & $3.5(0.5)$ & $3.4(1.0)$ \\
\hline Information on how to cope with other mental health problems & $3.4(0.7)$ & $3.4(0.8)$ \\
\hline The symptoms of substance use problems & $3.3(0.8)$ & $3.4(0.8)$ \\
\hline The effects of alcohol on women's health & $3.3(1.0)$ & $3.4(0.9)$ \\
\hline The telescoping course of substance use for women & $3.2(0.8)$ & $3.1(0.9)$ \\
\hline How the absence of a partner can influence recovery & $3.2(1.2)$ & $2.9(1.3)$ \\
\hline The effects of stimulants on women's health & $3.1(1.2)$ & $2.7(1.5)$ \\
\hline The effects of tobacco on women's health & $3.0(1.2)$ & $2.8(1.4)$ \\
\hline The effects of opioids on women's health & $2.8(1.5)$ & $2.7(1.6)$ \\
\hline The symptoms of eating problems & $2.8(1.5)$ & $2.6(1.5)$ \\
\hline The effects of marijuana on women's health & $2.5(1.3)$ & $2.5(1.5)$ \\
\hline $\begin{array}{l}\text { How to manage feelings and consequences of experiences } \\
\text { of abuse without using substances }\end{array}$ & - & $3.4(1.0)$ \\
\hline The importance of physical safety in relationships & - & $3.1(1.3)$ \\
\hline How the role of caretaker (or caregiver) impacts recovery & - & $3.1(1.2)$ \\
\hline The pros and cons of the caretaking (or caregiving) role & - & $3.0(1.1)$ \\
\hline
\end{tabular}




\section{Summary of prepilot results}

Results indicate that the intervention was acceptable and feasible for women in inpatient treatment for SUDs. Participants found gender-specific topics important to address in SUD treatment and rated the topics in the GSI as relevant. Women also reported high satisfaction with the intervention and suggested that improvements could be made by expanding the topics, adding additional "knowledge check" questions to enhance the interactivity, and increasing the visual appeal of the intervention.

\section{Pilot Study}

\section{Methods}

Participants. Participants for the pilot study $(n=60)$ were recruited using the same inclusion/exclusion criteria described above; however, recruitment was expanded to include women in inpatient, partial hospital, and outpatient treatment programs. Women in the outpatient program have the option of attending the in-person WRG; therefore, any women who were enrolled in this group were excluded from the pilot study, as they were already familiar with the material presented. Eighty-nine women were identified by clinicians as eligible for the study during the recruitment period: 60 (67\%) were enrolled, 17 (19\%) were not interested, too sick, or did not have time, $6(7 \%)$ were interested, but unable to schedule, 4 (4\%) were discharged before the RA could approach them, and $2(2 \%)$ were deemed ineligible (e.g., enrolled in WRG; unable to comprehend written material).

Intervention. Based on data from participants in the prepilot study, we made modifications to the GSI to expand the content and enhance interactivity. First, we added a module: Violence and Abuse: Getting Help. This change was made to address women's interest in receiving more information on trauma. At the end of the study, participants were also given a handout with resources on domestic violence and information on women and substance use. Second, we added a module: Women as Caretakers: Taking Care of Yourself While You are Taking Care of Others, to provide information on self-care and the role of relationships with others in recovery. We also infused information on depression into these two new modules to address participants' interest in having more information on depression and co-occurring psychiatric disorders. In addition, we increased the interactivity of each module by adding more "knowledge-check" and interactive questions (Fig. 3) throughout the GSI. Lastly, Living Stills (i.e., animated photos) of nature images were added to the conclusion of each module to increase visual appeal. The final revised GSI consisted of five modules in total.

\section{Results}

Demographics and background characteristics. The demographic characteristics for the pilot sample were very similar to the prepilot sample (Table 1). Fifty-eight percent of women were recruited from the inpatient detoxification unit, $22 \%$ from partial hospitalization, and $20 \%$ from outpatient treatment. Just under half the sample had children (47\%), with an average of 2.0 children ( $\mathrm{SD}=0.9$, range $1-4)$. Nineteen women (32\%) reported that they had a current intimate partner that used alcohol or drugs.
Table 4 provides information on psychiatric and substance use characteristics of the pilot sample. The most common cooccurring psychiatric problems experienced by participants in the past year were anxiety, depression, and PTSD; the most commonly reported substances of use were alcohol, tobacco, and opioids. Although $41 \%$ of the sample indicated that this was their first treatment episode, there was a wide range of previous treatment episodes. Very few women had ever participated in a women-only program for SUDs (13\%), but over half $(58 \%)$ had attended women-only self-help groups.

Gender-specific program assessment. Table 2 shows the ratings of importance for the 10 gender-specific topics assessed preintervention. As with the prepilot phase, the role of co-occurring psychiatric disorders in recovery and trauma and PTSD were rated highly. In addition, all participants rated counseling about healthy relationships as "very" (47\%) or "extremely" (53\%) important.

Completion and satisfaction. All the women completed all five modules of the GSI and accompanying assessments. On average, participants completed the GSI in 41.1 minutes $(\mathrm{SD}=$ 16.1); time spent per module ranged from 4.2 minutes ( $\mathrm{SD}=2.5$; Violence and Abuse: Getting Help) to 8.3 minutes $(\mathrm{SD}=4.1$; The Effects of Drugs and Alcohol on Women's Health). Mean ratings of the content areas ranged from 2.5 to 3.6 on a scale of $0-4$ (Table 3 ), indicating that each of the topics were rated as above average in relevance. The top two content areas were: the link between substance use and other mental health problems and the symptoms of anxiety; the lowest rated content area was the effects of marijuana on women's health.

Satisfaction with the intervention was high, with a mean of $34.5(\mathrm{SD}=4.7$, range $=22-40$, maximum score $=40)$, and did not differ by the level of care women were receiving $(F=1.2$, $\mathrm{df}=2, p=0.3)$ or prior experience in treatment $(t=0.1$, $\mathrm{df}=57, p=0.9)$, and was not correlated with age $(r=-0.1$, $p=0.5)$. The majority regarded the intervention as "somewhat" (5\%) or "extremely" $(91.7 \%)$ easy to use and "somewhat" to "extremely" visually appealing (98.3\%). All the women rated the gender-specific information as "a little", (10\%), "very" (31.7\%), or "extremely" (58.3\%) helpful.

Qualitative feedback from participants. Thirty-seven participants $(62 \%)$ provided qualitative feedback during the pilot study, with some providing feedback at multiple points: 11 participants (18\%) provided topic suggestions on the Relevance Questionnaire, 6 participants (10\%) included additional comments/suggestions on the CSQ, 9 participants (15\%) explained changes they would make to the program on the CSQ, and 30 participants $(50 \%)$ made verbal comments. Qualitative analyses followed the same procedures previously described for the prepilot study and responses were again grouped into "comments" or "suggestions." With regard to comments, four themes emerged:

(1) Learning new information: the most typical comment from participants was that the material was "interesting" or "helpful" because they learned new information. 


\section{*What can you do?*}

\section{Examine your past and present intimate partner relationships by asking.yourself the followingquestions:}

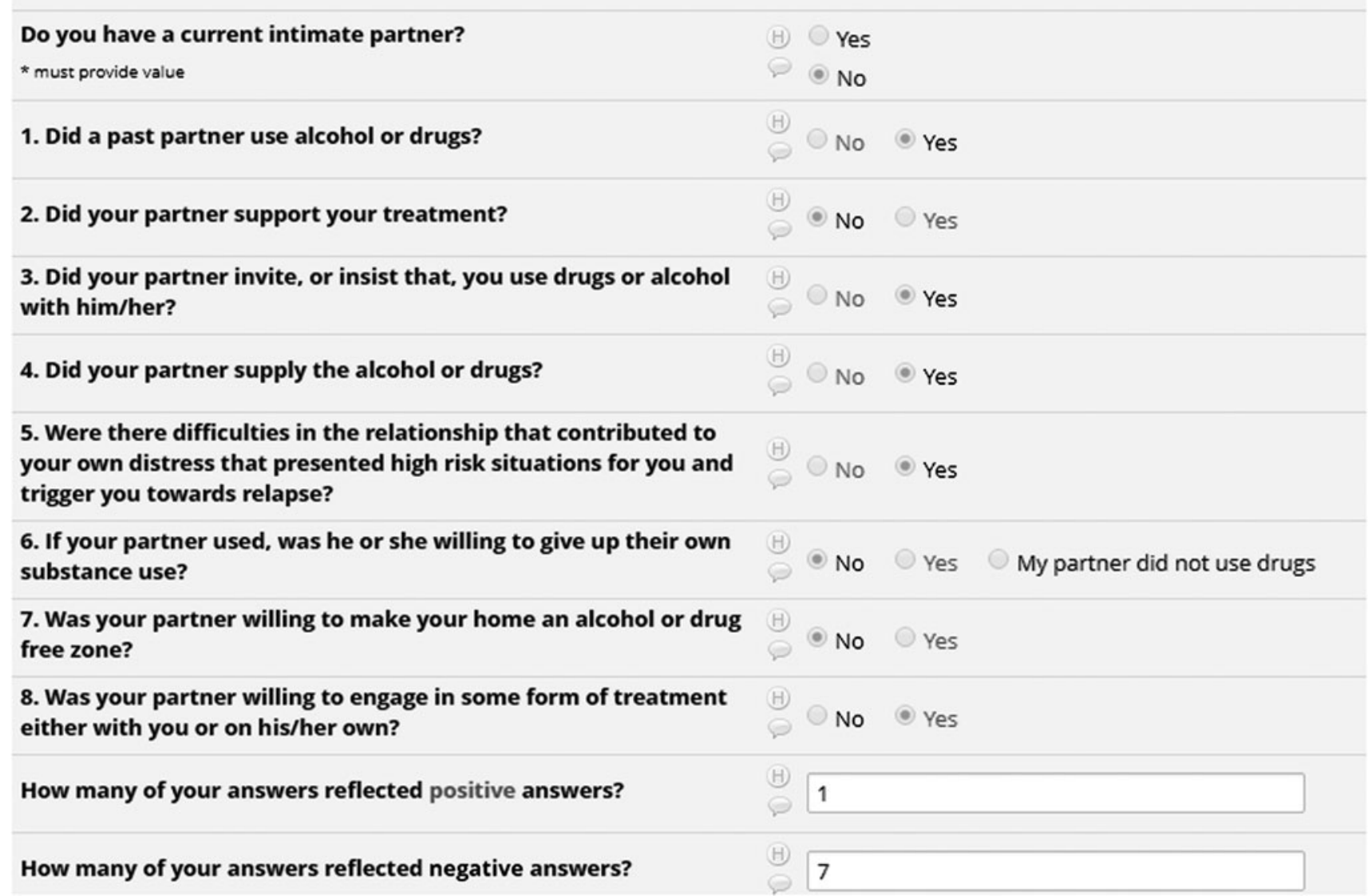

FIG. 3. Screenshot of interactive questions in the women and their partners module of the GSI.

"Very user friendly and I learned a lot of facts that will enable me to recover better both in treatment and after discharge and beyond."

(2) Relating to the information in the GSI: Similar to the prepilot study, women in the pilot study expressed a connection with the material. For example, while completing the module on Women and their Partners, one participant stated that "this was her." Another woman wrote:

"Beautiful...this hit home like no other program."

(3) The importance of the gender-specific information:

"[I] was surprised to learn about the telescoping effect. If women knew more about this effect, it might alleviate shame and guilt."

(4) The visual appeal of the GSI: several women commented that they thought the GSI was visually pleasing. In particular, women described the Living
Still images at the end of the modules as "beautiful" and "relaxing." One participant noted that she is a very visual person and "really liked learning through this program."

Three participants $(5 \%)$ expressed negative comments, including: the GSI was not geared for people who do not identify as someone with a SUD, not believing the information on marijuana was accurate, and not relating to the information on drug use (participant reported alcohol as the only problematic substance).

Suggestions for change were divided into three themes: (1) topic suggestions, (2) visual elements, and (3) technology solutions. Suggested topics included: age and isolation, problems with communication, navigating treatment while working, and changes in sexual drive that women experience in recovery. An additional suggestion was to include pets in 
Table 4. Pilot Sample Psychiatric and Substance Use Characteristics

\begin{tabular}{lr}
\hline & $\mathrm{n}=60$ \\
\hline Psychiatric problems, past year, $n(\%)$ & \\
Anxiety & $54(90.0)$ \\
Depression & $50(83.3)$ \\
Posttraumatic stress disorder & $25(41.7)$ \\
Attention deficit disorder & $23(38.3)$ \\
Eating disorder & $10(16.7)$ \\
Obsessive compulsive disorder & $10(16.7)$ \\
Bipolar disorder & $10(16.7)$ \\
Borderline personality disorder & $7(11.7)$ \\
Substances of use, lifetime, $n(\%)$ & \\
Alcohol & $45(75.0)$ \\
Tobacco & $24(40.0)$ \\
Opioids & $23(38.3)$ \\
Cocaine/crack & $16(26.7)$ \\
Sedatives & $14(23.3)$ \\
Cannabis & $10(16.7)$ \\
Hallucinogens, Phencyclidine (PCP) & $6(10.0)$ \\
Stimulants & $6(10.0)$ \\
Type of substance that has caused & \\
the most problems, past year, $n(\%)$ & \\
Alcohol & $34(56.7)$ \\
Drugs & $18(30.0)$ \\
Alcohol and drugs & $7(11.7)$ \\
First treatment episode for drugs or alcohol, & $24(40.7)$ \\
$n$ (\%) & \\
Number of previous treatment episodes & $2.0(4.6)$ \\
for alcohol problems, mean (SD) & \\
Range & $0-28$ \\
Number of previous treatment episodes & $2.5(7.5)$ \\
for drug problems, mean (SD) & $0-40$ \\
Range & \\
\hline
\end{tabular}

the Women as Caretakers module and provide in-depth information for those that want to learn more about each topic. Suggestions for visual element changes included bigger font and less text. Technology solution suggestions were to include an audio option to listen to the information and to allow users to connect directly from the GSI to resources on the internet.

\section{Summary of pilot results}

Results show that the five-module GSI was acceptable and feasible for women receiving SUD treatment across inpatient, partial hospitalization, and outpatient settings. Participants emphasized co-occurring psychiatric disorders, trauma, and relationships as important topics to address, and rated the topics in the GSI as relevant. Women suggested that the intervention could be enhanced by expanding the range and depth of the content, as well as providing alternative ways to engage with the GSI (i.e., audio, print).

\section{Discussion}

This study provides preliminary support that a genderspecific, web-based intervention is acceptable to women with SUDs and can be implemented in mixed-gender treatment settings at a range of levels of care. These results suggest that the intervention is applicable to women regardless of their age or previous experience in treatment and has the potential to be a highly sustainable strategy for increasing implementation of gender-specific treatment components across multiple levels of care.

Digital interventions have the potential to address some of the barriers of delivering gender-specific care in mixedgender SUD treatment programs. Given that there are more men than women in SUD treatment programs,${ }^{45}$ it is often difficult and costly to implement women-only groups. Inpatient programs provide an additional level of difficultly due to the short length of stay. The technological aspects of the GSI in both the prepilot and pilot studies were overwhelmingly rated as easy to use, visually appealing, and helpful, supporting the feasibility of delivering gender-specific psychoeducation in SUD treatment using a web-based platform.

Women in both samples expressed the importance of SUD treatment programs addressing co-occurring psychiatric disorders, trauma, and abuse. Moreover, the elements of the GSI that related to co-occurring mental health problems and the link between substance use and mental health problems were consistently rated as highly relevant. These findings are not surprising given the documented literature on the increased prevalence of co-occurring psychiatric disorders in women with SUDs, and the association of co-occurring disorders with treatment outcomes. ${ }^{2,46}$ The importance of integrated treatment that addresses both mental health and SUDs is also highlighted in the SAMHSA's guidance document for supporting women in co-ed settings. ${ }^{25}$

Although we did not quantitatively measure knowledge gained from the GSI, several women mentioned in their qualitative feedback that the information in the GSI was new to them. Given that most of the participants had been in treatment before participating in the study, it is significant that many of them had never received gender-specific information regarding their SUD. Moreover, the women indicated that learning the information would be helpful to their recovery.

This study is unique in that it is the first to examine genderspecific content in the absence of a single-gender environment. There is evidence that women in single-gender treatment environments report feelings of enhanced comfort, support, affiliation, and safety ${ }^{47,48}$; however, research also indicates that the single-gender environment alone was not sufficient to affect outcome. ${ }^{13}$ Given that most women are seeking care in mixed-gender treatment programs, it is essential to determine if gender-specific content delivered outside of the context of a single-gender environment is effective. This study shows that the gender-specific content was feasible to deliver via a webbased platform and women indicated that the GSI was helpful to them. The focus of these pilot studies was on feasibility and satisfaction; therefore, we did not investigate the effect of the GSI on substance use outcomes. It would be an important next step for future research to examine the effect of this intervention on women's engagement in treatment and treatment outcomes, including retention in treatment and substance use reductions.

There are several limitations to this study. First, the sample was racially and ethnically homogeneous, with the majority identifying as white and non-Hispanic or Latino. However, the clinical characteristics of the study participants are consistent with treatment-seeking women with SUDs with regard to substance use history, psychiatric comorbidity, and 
partnered and parenting status. In the pilot sample, anxiety, depression, and PTSD were the most commonly endorsed psychiatric problems, which is consistent with the literature on women with SUDs. ${ }^{2,7}$ Both study samples were also relatively well educated. It is possible that satisfaction ratings and experience of the GSI would differ in a sample with lower educational attainment, especially given the reliance on text-based information in the GSI. Further modifications should focus on reducing the amount of text and examining the GSI in a more diverse sample. A second limitation is the lack of data on participants who were eligible for the study but did not participate (40\% in prepilot and 33\% in pilot study). We do not have demographic data to compare characteristics based on participation status. Thus, it is possible that the sample who participated in the study are not representative of the general population of women in treatment for SUDs. Third, qualitative information was derived from survey questions plus verbal comments; however, we do not have qualitative data from all participants. To investigate the possibility that women who were more satisfied with the GSI were more likely to provide qualitative feedback, we compared satisfaction scores of participants who provided qualitative feedback to those who did not. No significant differences were found between these two groups in the prepilot or pilot studies. We also compared CSQ scores of participants based on whether or not they endorsed making changes to the GSI and found no significant differences in satisfaction in either study. Despite these limitations, the results of this study are promising for the feasibility of implementation and level of participant satisfaction with this gender-specific, web-based intervention for women with SUDs.

\section{Conclusions}

This brief gender-specific, web-based intervention has the potential to be a sustainable strategy for increasing exposure to a GSI across multiple levels of SUD treatment, even in settings with limited staff and financial capacity to offer specialty services.

\section{Acknowledgment}

The authors would like to gratefully acknowledge Brittany R. Iles, BS for her help with data collection.

\section{Author Disclosure Statement}

No competing financial interests exist.

\section{Funding Information}

Support for this article was provided by the Sarles Young Investigator Award for Research on Women and Addiction, McLean Hospital, and the Women's Mental Health Initiative and Innovation Funds, McLean Hospital.

\section{References}

1. Greenfield SF. Women and alcohol use disorders. Harv Rev Psychiatry 2002;10:76-85.

2. Greenfield SF, Back SE, Lawson K, Brady KT. Substance abuse in women. Psychiatr Clin N Am 2010;33:339-355.
3. Greenfield SF, Back SE, Lawson K, Brady KT. Women and addiction. In: Lowinson JH, Ruiz P, eds. Substance abuse: A comprehensive textbook, 5th ed. Philadelphia, PA: Lippincott, Williams and Wilkins, 2011, pp. 847-870.

4. Brady KT, Back SE, Greenfield SF. Women and addiction: A comprehensive handbook. New York: The Guilford Press, 2009.

5. Tang Z, Claus RE, Orwin RG, Kissin WB, Arieira C. Measurement of gender-sensitive treatment for women in mixed-gender substance abuse treatment programs. Drug Alcohol Depend 2012;123:160-166.

6. Greenfield SF, Grella CE. What is "women-focused" treatment for substance use disorders? Psychiatr Serv 2009; 60:880-882.

7. McHugh RK, Votaw VR, Sugarman DE, Greenfield SF. Sex and gender differences in substance use disorders. Clin Psychol Rev 2018;66:12-23.

8. Hser Y-I, Evans E, Huang D, Messina N. Long-term outcomes among drug-dependent mothers treated in womenonly versus mixed-gender programs. J Subst Abuse Treat 2011;41:115-123.

9. Prendergast ML, Messina NP, Hall EA, Warda US. The relative effectiveness of women-only and mixed-gender treatment for substance-abusing women. J Subst Abuse Treat 2011;40:336-348.

10. Kissin WB, Tang Z, Campbell KM, Claus RE, Orwin RG. Gender-sensitive substance abuse treatment and arrest outcomes for women. J Subst Abuse Treat 2014;46:332339.

11. Niv N, Hser Y-I. Women-only and mixed-gender drug abuse treatment programs: Service needs, utilization and outcomes. Drug Alcohol Depend 2007;87:194-201.

12. Claus RE, Orwin RG, Kissin W, Krupski A, Campbell K, Stark K. Does gender-specific substance abuse treatment for women promote continuity of care? J Subst Abuse Treat 2007;32:27-39.

13. Bride BE. Single-gender treatment of substance abuse: Effect on treatment retention and completion. Soc Work Res 2001;25:223-232.

14. Hien DA, Wells EA, Jiang $\mathrm{H}$, et al. Multisite randomized trial of behavioral interventions for women with cooccurring PTSD and substance use disorders. J Consult Clin Psychol 2009;77:607-619.

15. Linehan MM, Schmidt H, III, Dimeff LA, Craft JC, Kanter J, Comtois KA. Dialectical behavior therapy for patients with borderline personality disorder and drug-dependence. Am J Addict 1999;8:279-292.

16. Messina N, Calhoun S, Warda U. Gender-responsive drug court treatment: A randomized controlled trial. Crim Justice Behav 2012;39:1539-1558.

17. Slesnick N, Erdem G. Efficacy of ecologically-based treatment with substance-abusing homeless mothers: Substance use and housing outcomes. J Subst Abuse Treat 2013;45:416-425.

18. Epstein EE, McCrady BS, Hallgren KA, Cook S, Jensen NK, Hildebrandt T. A randomized trial of female-specific cognitive behavior therapy for alcohol dependent women. Psychol Addict Behav 2018;32:1-15.

19. Greenfield SF. Treating women with substance use disorders: The Women's Recovery Group manual. New York: Guilford Press, 2016.

20. Greenfield SF, Trucco EM, McHugh RK, Lincoln M, Gallop RJ. The Women's Recovery Group Study: A stage I trial of women-focused group therapy for substance use 
disorders versus mixed-gender group drug counseling. Drug Alcohol Depend 2007;90:39-47.

21. Greenfield SF, Sugarman DE, Freid CM, et al. Group therapy for women with substance use disorders: Results from the Women's Recovery Group Study. Drug Alcohol Depend 2014;142:245-253.

22. Substance Abuse and Mental Health Services Administration, Center for Behavioral Health Statistics and Quality. National survey of substance abuse treatment services $(\mathrm{N}$ SSATS): 2012. Data on substance abuse treatment facilities. Rockville, MD: Substance Abuse and Mental Health Services Administration, 2013.

23. Orwin RG, Francisco L, Bernichon T. Effectiveness of women's substance abuse treatment programs: A metaanalysis. Arlington, Virginia: Center for Substance Abuse Treatment, SAMHSA, 2001.

24. Grella CE, Greenwell L. Substance abuse treatment for women: Changes in the settings where women received treatment and types of services provided, 1987-1998. J Behav Health Serv Res 2004;31:367-383.

25. Substance Abuse and Mental Health Services Administration. Guidance document for supporting women in co-ed settings. Report no.: HHS publication no. (SMA) 16-4979. Rockville, MD: Substance Abuse and Mental Health Services Administration, 2016.

26. Santa Ana EJ, Martino S, Ball SA, Nich C, Frankforter TL, Carroll KM. What is usual about 'treatment-as-usual'? Data from two multisite effectiveness trials. J Subst Abuse Treat 2008;35:369-379.

27. Marsch LA. Leveraging technology to enhance addiction treatment and recovery. J Addict Dis 2012;31:313-318.

28. Litvin EB, Abrantes AM, Brown RA. Computer and mobile technology-based interventions for substance use disorders: An organizing framework. Addict Behav 2013;38:17471756.

29. Weiss RD, Jaffee WB, de Menil VP, Cogley CB. Group therapy for substance use disorders: What do we know? Harv Rev Psychiatry 2004;12:339-350.

30. Campbell AN, Nunes EV, Matthews AG, et al. Internetdelivered treatment for substance abuse: A multisite randomized controlled trial. Am J Psychiatry 2014;171:683-690.

31. Marsch LA, Guarino H, Acosta M, et al. Web-based behavioral treatment for substance use disorders as a partial replacement of standard methadone maintenance treatment. J Subst Abuse Treat 2014;46:43-51.

32. Bickel WK, Marsch LA, Buchhalter AR, Badger GJ. Computerized behavior therapy for opioid-dependent outpatients: A randomized controlled trial. Exp Clin Psychopharmacol 2008;16:132-143.

33. Carroll KM, Ball SA, Martino S, et al. Computer-assisted delivery of cognitive-behavioral therapy for addiction: A randomized trial of CBT4CBT. Am J Psychiatry 2008;165: 881-888.

34. Carroll KM, Ball SA, Martino S, Nich C, Babuscio TA, Rounsaville BJ. Enduring effects of a computer-assisted training program for cognitive behavioral therapy: A 6month follow-up of CBT4CBT. Drug Alcohol Depend 2009;100:178-181.

35. Baumann E, Czerwinski F, Reifegerste D. Gender-specific determinants and patterns of online health information seeking: Results from a representative German health survey. J Med Internet Res 2017;19:e92.

36. Bidmon S, Terlutter R. Gender differences in searching for health information on the internet and the virtual patient- physician relationship in Germany: Exploratory results on how men and women differ and why. J Med Internet Res 2015; $17:$ e156

37. Campbell AN, Nunes EV, Pavlicova M, et al. Gender-based outcomes and acceptability of a computer-assisted psychosocial intervention for substance use disorders. J Subst Abuse Treat 2015;53:9-15.

38. Ondersma SJ, Chase SK, Svikis DS, Schuster CR. Computer-based brief motivational intervention for perinatal drug use. J Subst Abuse Treat 2005;28:305-312.

39. Ondersma SJ, Svikis DS, Schuster CR. Computer-based brief intervention: A randomized trial with postpartum women. Am J Prev Med 2007;32:231-238.

40. Ondersma SJ, Svikis DS, Thacker LR, Beatty JR, Lockhart $\mathrm{N}$. Computer-delivered screening and brief intervention (e-SBI) for postpartum drug use: A randomized trial. J Subst Abuse Treat 2014;46:52-59.

41. Zanarini MC, Conkey LC, Temes CM, Fitzmaurice GM. Randomized controlled trial of web-based psychoeducation for women with borderline personality disorder. J Clin Psychiatry 2018;79.

42. Sadler AG, Mengeling MA, Torner JC, et al. Feasibility and desirability of web-based mental health screening and individualized education for female OEF/OIF reserve and national guard war veterans. J Trauma Stress 2013;26:401404.

43. Rotondi AJ, Anderson CM, Haas GL, et al. Web-based psychoeducational intervention for persons with schizophrenia and their supporters: One-year outcomes. Psychiatr Serv 2010;61:1099-1105.

44. Attkisson CC, Zwick R. The client satisfaction questionnaire: Psychometric properties and correlations with service utilization and psychotherapy outcome. Eval Program Plann 1982;5:233-237.

45. Substance Abuse and Mental Health Services Administration. Treatment episode data set (TEDS): 2000-2010. National admissions to substance abuse treatment services. Rockville, MD: Center for Behavioral Health Statistics and Quality, 2012.

46. Greenfield SF, Brooks AJ, Gordon SM, et al. Substance abuse treatment entry, retention, and outcome in women: A review of the literature. Drug Alcohol Depend 2007;86: $1-21$.

47. Greenfield SF, Cummings AM, Kuper LE, Wigderson SB, Koro-Ljungberg M. A qualitative analysis of women's experiences in single-gender versus mixed-gender substance abuse group therapy. Subst Use Misuse 2013;48: 750-760.

48. Sugarman DE, Wigderson SB, Iles BR, et al. Measuring affiliation in group therapy for substance use disorders in the Women's Recovery Group study: Does it matter whether the group is all-women or mixed-gender? Am J Addict 2016;25:573-580.

Address correspondence to:

Dawn E. Sugarman, PhD

Division of Alcohol and Drug Abuse

McLean Hospital

115 Mill Street

Belmont, MA 02478

E-mail: dsugarman@mclean.harvard.edu 